\section{THE SO-CALLED LATERAL ABERRANT THYROID}

BY

\author{
R. W. P. JOHNSON, M.B., Ch.B. \\ Consultant Pathologist
}

AND

\section{N. C. SAHA, M.B., B.S., M.R.C.P.Ed. Late Senior House Officer in Medicine Cuckfield Hospital, Cuckfield, Sussex}

Aberrant means "wandering," and thyroid tissue anywhere in the body outside the thyroid gland can be called aberrant. The term is now reserved for thyroid tissue found in the lateral part of the neck and does not include similar tissue at any other site-for example, in the midline of the neck in the course of the thyroglossal duct, struma ovarii, secondary deposits elsewhere in the body. The term "lateral aberrant thyroid" has been used since Albers (1829) used it to describe tumours containing thyroid tissue in the side of the neck in the region of the jugular vein. The tumours are often cystic and have a papillary pattern on histological examination. In 1896 Barker described a case of " cystic accessory thyroid," and since then a number of cases of lateral aberrant thyroid have been reported, but there has been considerable disagreement concerning the exact significance of the occurrence of thyroid tissue in the side of the neck. It is now generally agreed that this lateral aberrant thyroid tissue is always a secondary deposit from a primary carcinoma of the thyroid gland. There appears to be very little literature in this country about the condition, but one case was described by Murley (1950). In the last few years we have encountered six such cases, and this small series seems to be worthy of further study.

\section{Case Reports}

Case 1.-A youth aged 17, born in Argentina, had during childhood received deep $x$-ray therapy for an enlarged thymus gland. He went to his doctor in September, 1960, complaining of a chronic cough and was sent to the mass radiography unit for a chest $x$-ray examination. He was then referred to the chest physician on account of multiple bilateral opacities in the lungs. On examination he was fit and symptomless apart from a slight cough. His blood count was normal. The tuberculin test was positive $1 / 100$. Histoplasmin skin test was negative. A right scalene node biopsy was done, the sections showing papillary thyroid tissue. Lateral aberrant thyroid was diagnosed and it was thought that he had a primary carcinoma of the thyroid with multiple pulmonary metastases. Total thyroidectomy was performed and a solid tumour was felt in the lower pole of the right lobe of the gland. The growth was infiltrating the trachea and involving the right laryngeal nerve. As much as possible of the growth was removed. He made an uninterrupted recovery from the operation. The thyroid weighed $20 \mathrm{~g}$. and contained several small areas of calcification. Sections showed a primary papillary adenocarcinoma in the lower pole of the right lobe. There was also extensive fibrosis and lymphocytic infiltration of the gland. No malignant tissue was found in the left lobe. After radioactive iodine on two occasions the shadows in the lungs practically disappeared. Twelve months after operation he was perfectly fit.

Case 2.-A woman aged 27 gave a history that when 19 it was noticed by her mother that she had a small swelling below the angle of the jaw on the right side. There were no symptoms. In 1954 , at the age of 25 , she thought the swelling was getting larger and went to see her doctor. In 1956 a surgeon advised excision of the lump and she was admitted to hospital. A bilobed lymph node the size of a walnut was found near the top of the anterior triangle of the neck on the right side. This node was excised and sections showed papillary thyroid tissue. Radioiodine uptake tests were within normal limits. Total thyroidectomy was performed in April, 1956. The thyroid weighed $19 \mathrm{~g}$. There was a firm white nodule in the upper pole of the right lobe and two smaller similar nodules were in the lower part of the same lobe. The left lobe appeared normal. Sections showed a papillary adenocarcinoma of the right lobe with active lymphatic permeation and secondary deposits in the lymph nodes. Sections of the left lobe revealed similar areas of malignant thyroid tissue. She later went to America, and the latest report (July, 1961) stated that she had a small swelling in the neck, but this was not thought to be malignant.

Case 3.-A girl aged 18 came to hospital for a surgical opinion in 1960 . She complained of a lump in the left side of the neck that had been present for seven weeks. The lump was removed and sections showed a small area of cystic thyroid tissue with papillary proliferation. A diagnosis of lateral aberrant thyroid was made and total thyroidectomy was carried out. Sections of the thyroid showed a primary adenocarcinoma of the left lobe with similar malignant tissue in the right lobe. Chest $x$-ray films were normal and she has remained perfectly fit.

Case 4.-A girl aged 13 noticed a tender lump in the left side of her neck four weeks before coming to hospital in September, 1957. Enlarged lymph nodes were found on examination and there was a slight diffuse swelling of the thyroid gland. Chest $x$-ray films were clear. Lymph-node biopsy showed papillary thyroid tissue. At operation the whole thyroid gland was removed with block dissection of the glands in the left side of the neck. The thyroid weighed $29 \mathrm{~g}$. A small area of papillary adenocarcinoma was found in the left lobe. The growth extended towards the upper pole, where it was merged in a large neoplastic glandular mass. The patient remained well for four years but then developed secondary deposits in the chest.

Case 5.-A woman aged 26 had complained of swellings in the right side of the neck for two years. $X$-ray examination showed shadows in both lungs, and she was treated for tuberculosis. Improvement did not result and sarcoidosis was considered as a possible diagnosis. Biopsy of one of the lumps in the neck showed irregular thyroid follicles with a tendency to a papillary pattern. She was then treated for thyroid carcinoma.

Case 6.-A woman aged 42 was admitted to hospital on October 16, 1958, complaining of a lump in the right side of the neck. She had noticed the swelling five weeks previously when looking in the mirror, but there were no symptoms. On examination a lump could be felt in the right posterior triangle and a second nodule was found behind the sternomastoid muscle on the same side. One of the nodules was removed and sections showed cystic thyroid tissue. As a result of this finding, a total thyroidectomy was done on October 24 . The thyroid weighed $65 \mathrm{~g}$. Sections showed a clear-cell carcinoma with many cystic spaces. The gland was heavily infiltrated by lymphocytes. A month later no functioning thyroid tissue was demonstrable by radioactive iodine uptake tests.

\section{Discussion}

The thyroid gland is formed by the fusion of a medial anlage and two lateral anlages. The median anlage arises as a downgrowth from the primitive mouth, while the lateral anlages come from the fourth or fifth branchial arch. There have been several theories to explain the origin of thyroid tissue in the lateral part of the neck. Schrager (1906) suggested that portions of 
the lateral anlage became detached before fusion and that subsequent hyperplasia produced a benign tumour. For many years this theory was accepted, though a few authorities believed that lateral aberrant thyroid tissue represented a secondary deposit from a carcinoma in the homolateral lobe of the thyroid gland (Low, 1903). Crile (1939) thought the condition benign, but later changed his mind and stated that in all his 21 cases a primary carcinoma had been found in the thyroid (Crile, 1947).

It has been suggested that a lateral aberrant thyroid could be either a tumour arising in a laterally placed accessory thyroid or an epithelial inclusion in a lymph node similar to an adenolymphoma of the parotid gland, but it is now agreed that the condition is invariably a secondary carcinoma. The primary is often very small and cannot be detected clinically, but it can always be found by careful examination of the thyroid after surgical removal. The growth is usually papillary and often cystic; it is slow-growing and of a relatively low degree of malignancy. The thyroid often shows abnormal lymphocytic infiltration and areas of calcification. The six cases reported here confirm all of these points. Five patients presented with swellings in the side of the neck which biopsy showed to consist of papillary thyroid tissue. In Case 1 similar tissue was found in a scalene-node biopsy, though no lumps were palpable clinically. All the cases were found to have thyroid carcinomas. The primary growth is usually in the homolateral lobe of the thyroid, but small foci of carcinomatous tissue are quite often found in the opposite lobe (Cases 1 and 3).

The cases reported above have been treated by total thyroidectomy followed by radioactive iodine therapy for the distant metastases. All the patients were subsequently given desiccated thyroid by mouth. Dunhill (1937) described two cases of thyroid carcinoma in young people in whom recurrent growths disappeared on treatment with desiccated thyroid, and it has since been shown that many thyroid carcinomas are hormonedependent (Crile, 1957). If a patient becomes hypothyroid after treatment for cancer of the thyroid an excess of thyroid-stimulating hormone is produced by the pituitary, and this may stimulate the growth of the carcinoma. It is therefore important that all cases should be given adequate thyroid replacement therapy after thyroidectomy or treatment with radioactive iodine to suppress the production of thyroid-stimulating hormone.

This type of thyroid carcinoma can occur at any age, but it is important to note that it is often found relatively early in life. The age of onset in our cases ranged from 13 to 42 years, but cases have been found in children even younger. The secondary deposits are usually in the related lymph nodes and the growth may spread behind the carotid sheath, involving the recurrent laryngeal nerve. Metastases in the lungs are not uncommon, as in our Cases 1 and 5 . Case 1 had received $x$-ray treatment to the thymus gland in infancy, and it has been stated (Winship and Rosvoll, 1961) that a similar history can be obtained in $75-80 \%$ of cases of childhood cancer of the thyroid. It is interesting to note that Cases 1 and 5 were thought to be possible cases of sarcoidosis on account of the lung shadows, and Case 5 had been treated previously as nodular tuberculosis of the lungs. Winship and Rosvoll (1961) mention five cases which had been treated as tuberculosis.

\section{Summary}

Papillary thyroid tissue in the side of the neck is always a secondary deposit from a thyroid carcinoma, and the term lateral aberrant thyroid can be abandoned. Six cases of this condition, which often occurs in young people, are described. Distant metastases, particularly in the lungs, occur in some cases, but the prognosis is good. Clinically, a diagnosis of pulmonary tuberculosis or sarcoidosis may be made (Cases 1 and 5). After surgical removal of the thyroid or treatment with radioactive iodine it is essential to give full doses of thyroid to prevent the spread of hormone-dependent growths.

Our thanks are due to Dr. H. G. McGregor for permission to publish Cases 1, 2, and 3, and to Mr. P. A. Lane Roberts for Cases 4 and 6 ; Case 5 is under the care of Mr. Keith Powell.

\section{REFERENCES}

Albers, J. H. (1829). Die Pathologie und Therapie der Kehlkopfskrankheiten. Cnobloch, Leipzig.

Barker, A E. (1896). Lancet. 1, 102.

Crile, G. (1939). Surg. Gynec. Obstet., 69, 39.

Cr. (1947). Ibid., 85, 757.

- (1957). Cancer, 10, 1119.

Dunhill, T. (1937). Trans. med. Soc. Lond., 60, 234

Low, H C. (1903). Boston med. surg. J., 149, 616.

Murley, R. S (1950). Brit. J. Surg., 37, 324.
Schrager (1906). Surg. Gynec. Obstet., 3, 465.

Winship, T., and Rosvoll, R. V. (1961). Cancer, 14, 734.

\section{Preliminary Communications}

\section{Stimulation of Mammary Cancer by Prolactin and the Clinical Response to Hypophysectomy}

Hypophysectomy causes objective remission in nearly half the patients with advanced mammary cancer in whom it is undertaken. It does so, presumably, by removing the hormones which support the growth of the tumour. These include follicle-stimulating hormone (which controls the secretion of ovarian oestrogen) and corticotrophin (which controls that of adrenal oestrogen). The roles of growth hormone and of prolactin (mammotrophin) in mammary cancer are uncertain, although they are known to support the development and function of the normal breast.

The fact that the results of hypophysectomy are slightly better than those of adrenalectomy (Atkins et al., 1960) suggests that these or other unknown pituitary factors may be important. Some workers (Pearson and Ray, 1959) have reported that growth hormone stimulates tumour growth, but others (Lipsett and Bergenstal, 1960) have not found any such effect. There is little evidence that prolactin supports the growth of mammary cancer. Hadfield (1957) reported that prolactin was detected in the urine of three patients who subsequently responded favourably to hypophysectomy but not in that of four patients who failed to do so. Others (Baron et al., 1958) have not found any such association. Others again (Segaloff et al., 1951, 1953, 1954) have been unable to find any consistent relationship between the excretion of prolactin and the clinical response to various types of endocrine therapy. Lipsett and Bergenstal (1960) reported that prolactin of ovine origin did not increase the urinary excretion of calcium in patients with metastatic breast cancer. 